\title{
Impacto de una intervención de mejora de calidad sobre la incidencia de extubaciones no planeadas en una unidad de cuidados intensivos pediátricos
}

\author{
Impact of a quality improvement intervention on the incidence \\ of unplanned extubation in a Pediatric Intensive Care Unit
}

\author{
Dra. Claudia N. Meregallia, Dr. Facundo A. Jorro Barón ${ }^{a}$ Lic. Kga. Marcela A. \\ $D^{\prime}$ Alessandro $^{a}$, Lic. Kgo. Esteban P. Danzi ${ }^{a}$ y Dr. Gustavo E. Debaisia
}

\section{RESUMEN}

Introducción. Las extubaciones no planeadas (ENP) son eventos adversos frecuentes y prevenibles de la ventilación mecánica.

Objetivos. Conocer las causas de las extubaciones no planeadas y evaluar la utilidad de la aplicación de una intervención de mejora de la calidad para disminuir ese evento.

Población. Pacientes con ventilación mecánica invasiva durante más de 12 horas durante el período comprendido entre el 1 de mayo del 2010 y el 30 de abril del 2011 en una unidad de cuidados intensivos pediátricos (UCIP).

Diseño. Estudio antes-después no controlado. Se aplicó una intervención de mejora de calidad en el período noviembre de 2010-abril de 2011 que incluyó capacitación del personal de la UCIP, estandarización de la fijación del tubo endotraqueal y control de la fijación.

Resultados. Ingresaron en la UCIP 395 pacientes, $262(66 \%)$ recibieron ventilación mecánica durante más de 12 horas. Presentaron 30 episodios de ENP 27 pacientes: 22 durante el período mayo a octubre de 2010, y 8 durante el período noviembre de 2010-abril de 2011. Las causas más frecuentes fueron la incorrecta fijación del tubo endotraqueal $(n=$ 11) y la realización de un procedimiento de atención y cuidado $(n=11)$. Los pacientes que la presentaron requirieron más días de ventilación mecánica: 7 (3 a 15,5) contra 5 (2 a 8) días; $p=$ 0,077 y de internación: 9,5 (5 a 19) contra 7 ( 4 a 10) días; $p=0,049$. Durante la intervención de mejora de la calidad la incidencia media de ENP disminuyó de 2,30 (IC 95\% 1,12 a 3,49) a 0,86 (IC $95 \%$ 0,27 a 1,44) por 100 días de ventilación mecánica $(p=0,037)$.

Conclusiones. Las causas más frecuentes de ENP fueron la incorrecta fijación del tuboendotraqueal y la realización de procedimientos de atención y cuidado. La implementación de una intervención de mejora de la calidad disminuyó la incidencia de ENP.

Palabras clave: extubación no planeada, niños, extubación traqueal, mejora de la calidad, ventilación mecánica.

http:/ /dx.doi.org/10.5546/aap.2013.391

\section{INTRODUCCIÓN}

Como consecuencia del desarrollo de técnicas de soporte vital para el tratamiento de los niños críticamente enfermos, la intubación endotraqueal y la ventilación mecánica (VM) se han transformado en prácticas rutinarias en la UCIP. En la mayoría de los casos, el tubo endotraqueal (TET) se retira en forma electiva cuando la causa que dio inicio a la VM está resuelta. Un evento adverso frecuente, y muchas veces prevenible de la VM, es la extubación no planeada (ENP), la cual se define como la salida del TET en un momento distinto del elegido para la extubación electiva. ${ }^{1}$ La ENP aumenta la morbimortalidad de los pacientes de la UCIP y los expone a las complicaciones de la intubación endotraqueal de emergencia cuando se requiere la reintubación, prolongando el tiempo de VM y de permanencia en la UCIP. ${ }^{2-6}$

Los programas de mejora continua de la calidad para disminuir la incidencia de las ENP han demostrado que, a través de la capacitación del personal de la UCIP, la identificación de los pacientes de riesgo, la estandarización de procedimientos de atención y cuidado, y el desarrollo de protocolos de sedación y analgesia y destete de la VM, es posible disminuir la incidencia de ENP. ${ }^{1,2,7-10}$

Los objetivos del presente estudio fueron conocer las causas de las ENP para diseñar una intervención de mejora de la calidad destinada a disminuir su incidencia y evaluar la eficacia de esa intervención. 


\section{MATERIALES Y MÉTODOS Diseño}

Estudio antes-después no controlado.

\section{Descripción del ámbito de estudio}

La investigación se realizó en la UCIP del Hospital General de Niños "Pedro de Elizalde". Esta unidad cuenta con 11 camas médicoquirúrgicas, sin cirugía cardiovascular. La vía de intubación orotraqueal fue utilizada con mayor frecuencia. La técnica de fijación hasta la intervención no estaba normatizada y generalmente se realizaba con cinta adhesiva de seda o común, colocada en forma simple o doble sobre el labio superior. Todos los pacientes en VM invasiva se mantuvieron sedados con fentanilo y midazolam en goteo continuo; en caso de requerirse, se utilizaban bloqueantes neuromusculares (vecuronio o pancuronio). La unidad no contaba con protocolo de sedoanalgesia ni de destete de la VM durante el estudio.

\section{Muestra}

Todos los niños que requirieron intubación endotraqueal para VM invasiva durante más de 12 horas en la UCIP durante el período comprendido entre el 1 de mayo de 2010 y el 30 de abril de 2011.

Se calculó un número mínimo de 800 días de VM por grupo para poder detectar una diferencia $(p<0,05)$ en la tasa de ENP con una potencia estadística mayor del $80 \%$, tomando en cuenta una tasa de referencia $<1$ ENP por 100 días de VM y una incidencia antes de la intervención $>2$ ENP por 100 días de VM.

\section{Instrumento de recolección de datos}

Los datos se obtuvieron por observación directa de los eventos. Para evitar la pérdida de eventos y de datos en el momento de la ENP, se revisaron diariamente las hojas de evolución y el libro de informes de enfermería.

La ficha de recolección de datos presentó tres partes (ver Anexo 1 en páginas electrónicas).

Se definió como ENP a la salida del TET en un momento distinto del elegido para la extubación electiva. ${ }^{1}$ La incidencia se expresó en número de ENP por 100 días de VM. Se consideraron causa de ENP y su correspondiente definición:

1) Realización de procedimientos de atención y cuidado: se incluyeron la higiene, el cambio de fijación del TET, la aspiración de secreciones, la realización de una radiografía, el cambio de decúbito y la colocación de accesos vasculares.

2) Fijación incorrecta de TET: tela adhesiva no tensa o despegada que permitía el desplazamiento del TET. La fijación del TET es un procedimiento de atención y cuidado del paciente crítico; se consideró separada del resto de los procedimientos por su importancia como causa de ENP.

3) Inadecuado nivel de sedación: se evaluó con el puntaje de Ramsay. Se consideró inadecuado nivel de sedación a aquel paciente con puntaje de nivel 1, y adecuado, entre los niveles 2 y $6 .{ }^{11}$

4) Inadecuada relación enfermero-paciente: cualquier relación mayor de 1:2.

5) Secreciones abundantes: más de tres aspiraciones por turno de enfermería de 6 horas.

6) Destete de la VM: transición entre el soporte ventilatorio y la respiración espontánea completa. ${ }^{12}$

7) Transporte: ocurrida durante el traslado al quirófano o a la sala de hemodinamia, o para efectuar un estudio de diagnóstico por imágenes.

La investigación se desarrolló en dos etapas. En la primera etapa ("antes"), comprendida entre mayo y octubre de 2010, se analizaron los datos basales. En la segunda etapa ("después"), comprendida entre noviembre de 2010 y abril de 2011, se aplicó una intervención de mejora de la calidad para disminuir la incidencia de ENP en la unidad.

Paquete de medidas aplicado (ver Anexo 2 en páginas electrónicas):

- Capacitación del personal de la UCIP con la finalidad de:

- Comunicar los resultados de la primera etapa de la investigación.

- Concientizar sobre la importancia y las consecuencias de la ENP.

- Difundir el contenido y la forma de implementación del paquete de medidas de mejora.

- Estandarización de la fijación del TET. Se estandarizó la técnica de fijación y el material usado (Tensoplast Sport ${ }^{\circledR}$ ); también se registró, en una ficha ubicada a la cabecera del paciente, la información sobre la profundidad y el tamaño del TET.

- Estandarización del monitoreo de la fijación del TET. Se verificó la correcta fijación del TET una vez por turno de enfermería y antes de la higiene del paciente.

\section{Análisis estadístico}

Los datos se analizaron con el programa STATA 10.1 (Statistics/Data Analysis Stata 
Corporation 4905 Lakeway Drive College Station, TX, EE.UU.). Los valores se expresaron como media y desviación estándar o mediana y rango intercuartil, según correspondiera.

Los grupos se compararon utilizando la prueba de la $t$ de Student o la prueba de Wilcoxon según normalidad. Para las variables dicotómicas se utilizó la prueba de la $\chi^{2}$. Se consideró significativo un valor de $p<0,05$.

Para la comparación gráfica de la incidencia de ENP entre los dos períodos se utilizó un $u$-control chart, donde se tomó como límite de control superior e inferior \pm 2 desviaciones estándar.

\section{RESULTADOS}

Durante la primera etapa de la investigación ingresaron en la UCIP del HGNPE 232 pacientes, de los cuales $162(70 \%)$ recibieron VM durante más de 12 horas. El número total de días de VM fue 1026. Se registraron 22 episodios de ENP en 20 pacientes: 18 pacientes presentaron una y 2 , dos ENP. La tasa media de incidencia de ENP fue de 2,30 por 100 días de VM.

Se identificaron como causas más frecuentes la incorrecta fijación del TET $(n=11)$ y la realización de un procedimiento de atención y cuidado $(n=$ 11) (Figura 1).

Sólo en 9 ENP se identificó una causa única; en las 13 restantes las causas fueron múltiples.

Los procedimientos de atención y cuidado vinculados a las ENP fueron: la higiene $(n=5)$, la realización de una radiografía $(n=1)$, la colocación de un acceso vascular $(n=1)$, el cambio de fijación del TET $(n=1)$ y no se registró $(n=3)$.

El mayor porcentaje (41\%) ocurrió durante el turno mañana de enfermería de los días hábiles (Figura 2).

Los pacientes con ENP requirieron más días en VM: 7 (IQ 3-15,5) contra 5 (IQ 2-8) días, $p=0$ 0,077; y permanecieron más días internados en la UCIP: 9,5 (IQ 5-19) contra 7 (IQ 4-10) días, $p=0,049$. No se registraron muertes asociadas a la ENP.

De las 22 ENP, $16(73 \%)$ requirieron reintubación. Todas las que ocurrieron durante el soporte ventilatorio completo la necesitaron, mientras que de las que sucedieron durante el destete de la VM solo 1 requirió reintubación.

El 68\% $(n=15)$ de las ENP se produjeron durante el soporte ventilatorio completo y el $32 \%(n=7)$, durante el destete de la ventilación mecánica; $68 \%$ ocurrieron entre el día 1 y 5 del inicio de la VM. Los grupos de pacientes que presentaron ENP y los que no la presentaron fueron similares en edad, peso e índice pediátrico de mortalidad II (Pediatric index of mortality, PIM) (Tabla 1).

Durante la aplicación de la intervención de mejora de la calidad, ingresaron en la unidad 163 pacientes, de los cuales 100 (61\%) requirieron VM por más de 12 horas. Los días totales de VM en el período fueron 847 . Se registraron 8 episodios de ENP en 7 pacientes. Se identificaron como causas de las ENP: el destete de la VM $(n=5)$, la inadecuada relación E:P $(n=4)$, el inadecuado

FIgURA 1. Causas de extubación no planeada (ENP)

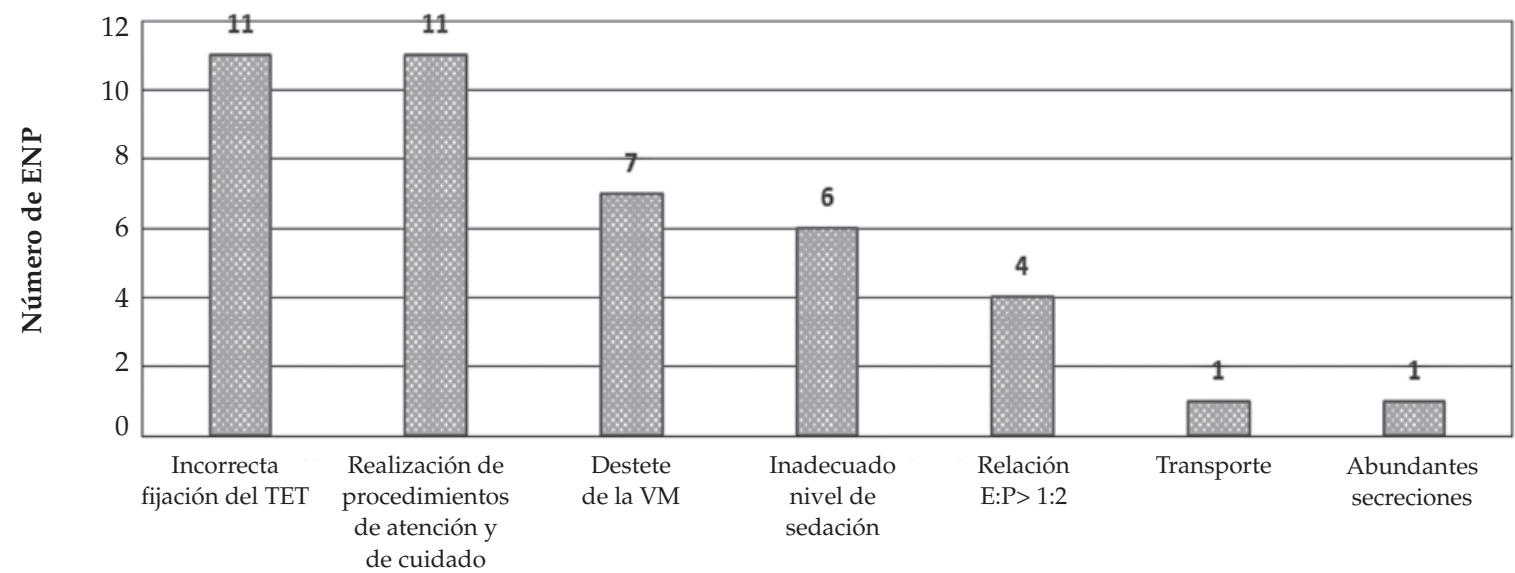

Causas de ENP

TET: Tubo endotraqueal

VM: Ventilación mecánica

E:P: Enfermera: paciente 
nivel de sedación $(n=4)$, la incorrecta fijación del TET $(n=3)$, y la realización de procedimientos de atención y cuidado $(n=1)$ La tasa media de incidencia de ENP fue 0,86 por 100 días de VM, siendo menor a la tasa media de incidencia antes de la implementación de la intervención de mejora de la calidad (2,30; IC 95\% 1,12 a 3,49 contra 0,86 ; IC $95 \% 0,27$ a $1,44, p=0,037$ ) (Figura 3). Los pacientes antes y después de la aplicación de la estrategia de mejora fueron similares excepto por el PIM II, el cual fue mayor para los pacientes que ingresaron en la segunda etapa de la investigación (3,6; IQ 1,3 a 8,4 contra 7; IQ 2,7 a 16,2, p=0,0007) (Tabla 2).

La mortalidad de los pacientes que requirieron VM por más de 12 horas durante todo el período de estudio fue de $9,5 \%(n=25)$.

\section{DISCUSIÓN}

Las ENP son eventos adversos frecuentes y muchas veces prevenibles de la VM. Su incidencia en la población pediátrica en los estudios publicados en los últimos 20 años varía de 0,11 a 2,7 ENP por 100 días de VM. ${ }^{3,5}$

La incidencia observada en la UCIP del HGNPE durante los primeros 6 meses de estudio fue de 2,30 por 100 días de VM, similar a la informada por otros estudios en la etapa previa a la aplicación de una intervención para disminuir su ocurrencia., 2,4,7,13 No hay consenso acerca de la incidencia aceptable de ENP. Una tasa igual a cero puede ser deseable, pero al mismo tiempo, peligrosa. El objetivo se podría lograr utilizando altas dosis de sedación o extubando a los pacientes antes de que cumplan con los criterios de destete de la VM,

FIGURA 2. Distribución de las extubaciones no planeadas en los turnos de enfermería $(n=22)$

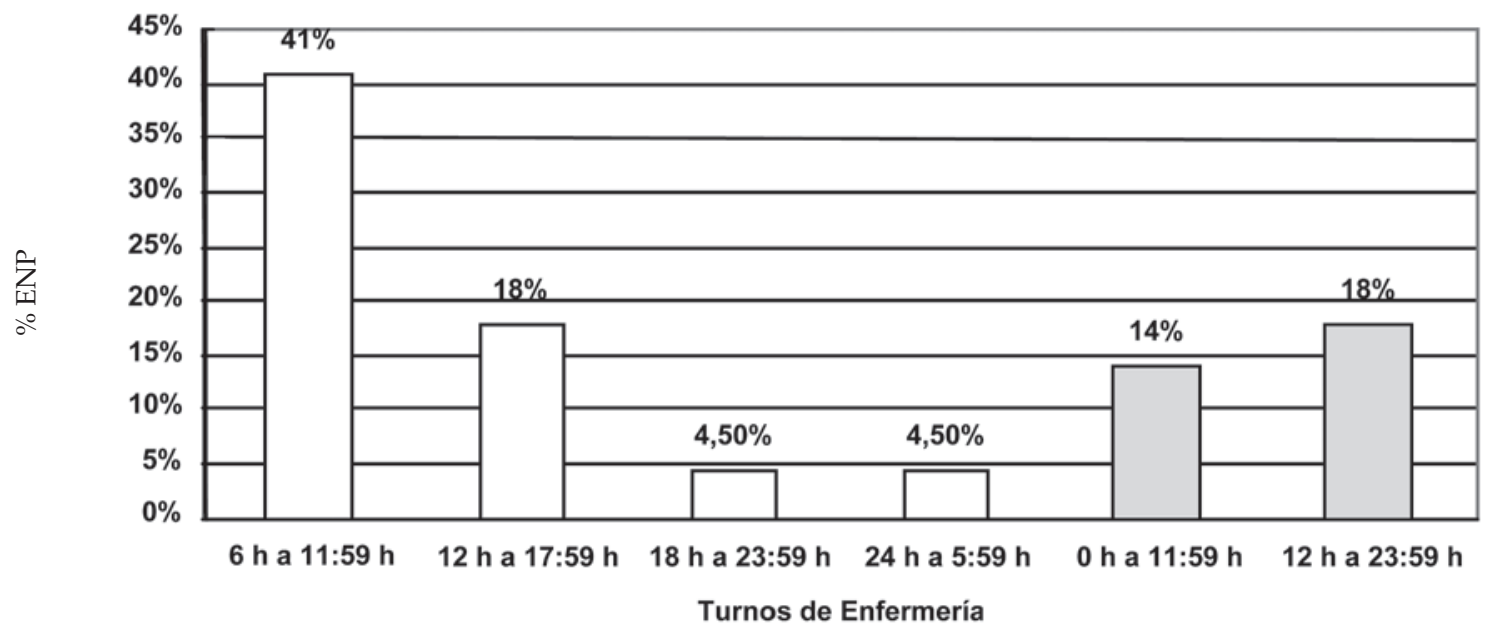

Lunes a viernes

Sábados, domingos y feriados

TABla 1. Características demográficas y clínicas de la población en estudio

\begin{tabular}{lccc}
\hline & $\begin{array}{c}\text { Pacientes sin ENP } \\
(\mathbf{n}=\mathbf{1 4 2})\end{array}$ & $\begin{array}{c}\text { Pacientes con ENP } \\
(\mathbf{n}=\mathbf{2 0})\end{array}$ & $\mathbf{p}$ \\
\hline Edad (meses), mediana (IQ) & $7(3-26)$ & $12(2-50)$ & 0,888 \\
Peso (kg), mediana (IQ) & $7,35(5-12)$ & $8,8(5,3-12)$ & 0,643 \\
PIM II, mediana (IQ) & $3,3(1,22-8,4)$ & $5,84(1,3-8,5)$ & 0,543 \\
Estadía en UCIP (días), mediana (IQ) & $7(4-10)$ & $9,5(5-19)$ & $\mathbf{0 , 0 4 9}$ \\
Días de VM, mediana (IQ) & $5(2-8)$ & $7(3-15,5)$ & 0,077 \\
Sexo femenino, número (\%) & $57(40 \%)$ & 0,402 \\
\hline
\end{tabular}

PIM II: índice pediátrico de mortalidad II; VM: ventilación mecánica; UCIP: unidad de cuidados intensivos pediátricos; ENP: extubaciones no planeadas. 
situaciones vinculadas a mala evolución clínica, requerimientos de mayor número de días de VM y de internación en la UCIP.

Las causas asociadas a ENP comprenden la edad del paciente, la agitación y el nivel de sedación, la fijación del TET, la cantidad de secreciones, la relación enfermero-paciente, la vía de intubación endotraqueal, la causa de ingreso en la UCIP, la contención física, la realización de procedimientos de atención y cuidado, el transporte, y el destete de la VM. ${ }^{1-3,14}$ No se incluyeron en el análisis de las causas de ENP

FIGURA 3. Incidencia de extubaciones no planeadas antes y después de la implementación de la estrategia de mejora

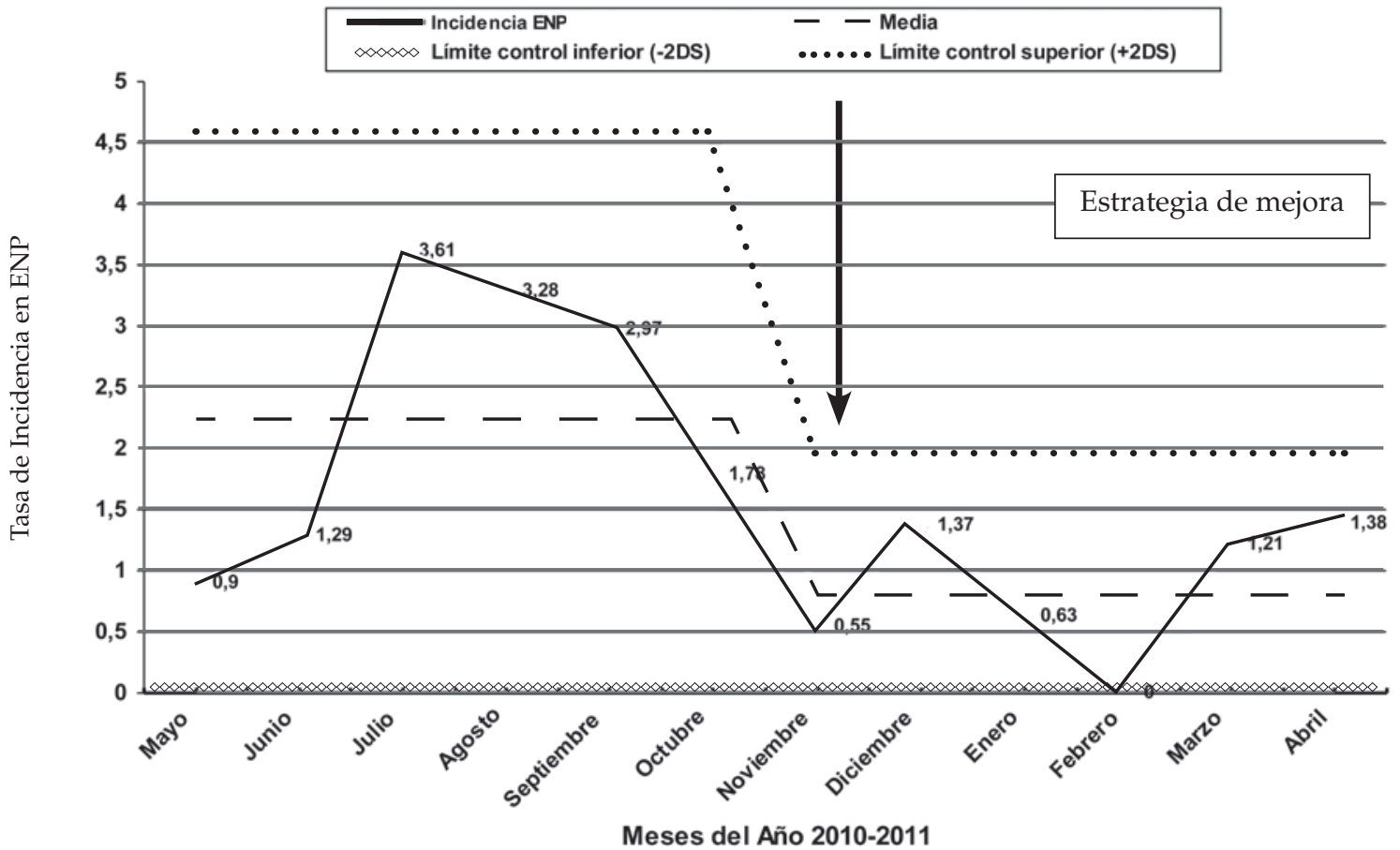

TABLA 2. Características demográficas de los pacientes que ingresaron en la UCIP y requirieron ventilación mecánica por más de 12 horas antes y después de la implementación de la estrategia de mejora

\begin{tabular}{lccc}
\hline & $\begin{array}{c}\text { Antes } \\
(\boldsymbol{n = 1 6 2})\end{array}$ & $\begin{array}{c}\text { Después } \\
(\boldsymbol{n}=\mathbf{1 0 0})\end{array}$ & $p$ \\
\hline Edad (meses), mediana (IQ) & $7(1-27)$ & $9(4-33,5)$ & 0,189 \\
Peso (kg), mediana (IQ) & $7,85(5-12)$ & $8,4(5,1-13)$ & 0,429 \\
Sexo femenino (\%) & 41,4 & 49 & 0,226 \\
PIM II, mediana (IQ) & $3,6(1,3-8,4)$ & $7(2,7-16,2)$ & $\mathbf{0 , 0 0 0 7}$ \\
Días de VM, mediana (IQ) & $5(2-8)$ & $5(2-8,5)$ & 0,983 \\
Estadía en UCIP (días), & $7(4-11)$ & $7,5(4-14,5)$ & 0,330 \\
mediana (IQ) & & & $\mathbf{0 , 0 3 7}$ \\
Tasa de incidencia de ENP, & $2,30(1,12-3,49)$ & $0,86(0,27-1,44)$ &
\end{tabular}

PIM II: índice pediátrico de mortalidad II; VM: ventilación mecánica; UCIP: unidad de cuidados intensivos pediátricos; IQ: intervalo intercuartil; IC 95\%: intervalo de confianza del 95\%. 
la vía de intubación endotraqueal, dado que en el período de estudio en todos los pacientes se utilizó la vía orotraqueal; la utilización de sujeciones, por no contar con evidencia científica a favor de su utilización; y la causa de ingreso en la UCIP, ya que la mayoría de los autores coinciden en que la menor incidencia de ENP en los pacientes con causa de ingreso en la UCIP quirúrgica se debe al menor número de días de VM. Las causas asociadas a las ENP más frecuentes en nuestra unidad fueron la inadecuada fijación del TET y la realización de procedimientos de atención y cuidado, entre los cuales la higiene del paciente fue la predominante.

La edad de los pacientes se ha considerado un factor de riesgo para ENP. ${ }^{2,7}$ En nuestra unidad, la edad de los pacientes que presentaron ENP no mostró diferencias estadísticamente significativas con respecto a la edad de los que no la presentaron.

Hay varias posiciones con respecto a los momentos del día en los cuales ocurren más frecuentemente las ENP. Algunos autores sostienen que no hay diferencias entre el día y la noche, otros afirman que son más frecuentes durante el día, porque se les realizan más procedimientos a los pacientes, o durante la noche, por el menor nivel de vigilancia. $2,6,7,14-16$ En nuestra unidad la mañana es el momento en el cual se produjeron mayor número de ENP $(41 \%)$, coincidiendo con la realización de la mayor cantidad de procedimientos de atención y cuidado. La higiene se realizaba de forma rutinaria en el turno mañana de enfermería (de 6:00 a 11:59 h).

En esta investigación, las ENP prolongaron los días de VM, si bien la diferencia no fue significativa, e incrementaron los días de estadía en la UCIP, sin aumentar la mortalidad. 2,3,7,11,14-19

La incidencia de reintubación en los distintos estudios se encuentra entre el 33,3\% y el 78\%.2,3,7,15,17,18,20,21 En la bibliografía se ha considerado que las ENP ocurridas durante el destete de la VM han tenido menos posibilidades de requerir nueva intubación que las ocurridas durante el soporte ventilatorio completo. ${ }^{7,11,18}$ En nuestra unidad la incidencia de reintubación fue alta y esto se debe a que la mayoría de las ENP ocurrieron durante el soporte ventilatorio completo, en pacientes sin resolución de la causa que motivó la VM o con nivel de sedación inadecuado para sostener la ventilación espontánea.
La implementación de programas de mejora continua de la calidad, que incluyan la capacitación del personal, la mejora de la comunicación entre los profesionales, la estandarización de los procedimientos de atención y cuidado, la incorporación de protocolos de destete de la VM y de sedoanalgesia, y la identificación de los pacientes de mayor riesgo de padecer ENP han mostrado disminuir la incidencia de ENP. . $^{2,7,9,10}$ En la población pediátrica se encontraron pocos estudios que evalúen estrategias de mejora para disminuir su incidencia. ${ }^{2,4,7,10}$ Da Silva y cols. ${ }^{2}$ implementaron un programa de mejora continua de la calidad destinado a disminuir la incidencia de ENP que incluía: capacitación del personal, estandarización de procedimientos, identificación de los pacientes de riesgo, y realización de un protocolo de sedación y analgesia. Tras su aplicación, la incidencia de ENP disminuyó de 2,9 por 100 días de VM a 0,6 por 100 días de VM $(p=0,0001)$. Rachman y cols., ${ }^{4}$ luego de incorporar un programa de mejora continua que incluía la capacitación del personal, la estandarización de la fijación del TET y el monitoreo de la fijación del TET, también observaron una disminución de la incidencia de ENP de 6,4 por 100 días de VM a 1 por 100 días de VM $(p=0,04)$.

Por su parte, Sadowski y cols. ${ }^{7}$ aplicaron un programa de mejora que incluía: capacitación del personal, protocolo de destete de la VM y protocolo de sedoanalgesia para pacientes en VM; durante su aplicación la incidencia de ENP disminuyó de 1,6 por 100 días de VM a 0,6 por 100 días de VM.

Durante la implementación de la intervención de mejora de la calidad la incidencia de ENP disminuyó un $63 \%$, coincidiendo con la bibliografía. ${ }^{2,4,7,15}$

Las limitaciones de esta investigación son, en primer lugar, que es un estudio de centro único. En segundo lugar, que no se puede descartar el cambio de comportamiento del personal que participó en la segunda parte de la investigación, debido a la conciencia de ser observado activamente (efecto Hawthorne). En tercer lugar, la mayor gravedad de los pacientes en la segunda etapa de la investigación podría haber contribuido a la disminución de la incidencia de las ENP, ya sea por la mayor vigilancia del paciente o por la menor capacidad de este de provocar una autoextubación. Por lo tanto, no existe certeza de que los resultados 
estén asociados a la intervención aplicada, dada la ausencia de un grupo de control. Finalmente, el estudio no evalúa la persistencia en el tiempo del beneficio hallado.

\section{CONCLUSIONES}

Las causas más frecuentes de ENP en la UCIP del HGNPE fueron la incorrecta fijación del TET y la realización de procedimientos de atención y cuidado. La utilización de una intervención de mejora de la calidad, consistente en la capacitación del personal de la UCIP y en la estandarización de la fijación y del monitoreo de la fijación del tubo endotraqueal, disminuyó la incidencia de ENP.

\section{BIBLIOGRAFÍA}

1. Da Silva PSL, de Carvalho WB. Unplanned extubation in pediatric critically ill patients: A systematic review and best practice recommendations. Pediat Crit Care Med 2010;11(2):287-94.

2. Da Silva PSL, de Aguilar VE, Neto HM, de Carvalho WB. Unplanned extubation in a pediatric intensive care unit: Impact of a quality improvement programme. Anaesthesia 2008;63(11):1209-16.

3. Piva JP, Amantéa S, Luchese S, Giugno K, et al. Extubação acidental em uma unidade de terapia intensiva. J Pediatr (Río J) 1995;71(2):72-6.

4. Rachman BR, Watson R, Woods N, Mink RB. Reducing unplanned extubations in pediatric intensive care unit: A systematic approach. Int J Pediatr 2009;2009:820495.

5. Little LA, Koening JC Jr, Newth CJ. Factors affecting accidental extubations in neonatal and pediatric intensive care. Crit Care Med 1990;18(2):163-5.

6. Curry K, Cobb S, Kutash M, Diggs C. Caracteristics associated with unplanned extubations in a surgical intensive care unit. Am J Crit Care 2008;17(1):45-51.

7. Sadowski R, Dechert RE, Bandy KP, JunoJ, etal. Continuous quality improvement: Reducing unplanned extubations in pediatric intensive care unit. Pediatrics 2004;114(3):628-32.

8. Richmond AL, Jarog DL, Hanson VM. Unplanned extubation in adult critical care: Quality improvement and education payoff. Crit Care Nurse 2004;24(1): 32-7.

9. Chiang AA, Lee KC, Lee JC, Wei CH. Effectiveness of a continuous quality improvement program aiming to reduce unplanned extubation: A prospective study. Intensive Care Med 1996;22(11):1269-71.

10. Popernack ML, Thomas NJ, Lucking SE. Decreasing unplanned extubations: Utilization of the Penn State Children's Hospital sedation algorithm. Pediatr Crit Care Med 2004;5(1):58-62.

11. Hartaman ME, Macrory DC, Schulman SR. Efficacy of sedation regimens to facilitate mechanical ventilation in the pediatric intensive care unit. A systematic review. Pediatr Crit Care Med 2009;10(2):246-55.

12. Newth CJL, Venkataraman S, Wilson DF, Meert KL, et al. Weaning and extubation readiness in pediatric patients. Pediatr Crit Care Med 2009;10(1):1-11.

13. SesslerCN. Unplanned extubations: Making progress using CQI. Intensive Care Med 1997;23(2):143-5.

14. Ream RS, Mackey K, Leet T, Green MC, et al. Association of nursing workload and unplanned extubations in a pediatric intensive care unit. Pediatr Crit Care Med 2007;8(4):366-71.

15. Bouza C, Garcia E, Diaz M, Segovia E, Rodríguez I. Unplanned extubation in orally intubated medical patients in the intensive care unit: A prospective cohort study. Heart Lung 2007;36(4):270-6.

16. Coppolo DP, May JJ. Self-extubations: A 12-month experience. Chest 1990;98(1):165-9.

17. Atkins PM, Mion LC, Mendelson W, Palmer RM, et al. Characteristics and outcome of patients who self-extubate from ventilatory support: a case-control study. Chest 1997;112(5):1317-23.

18. Phoa LL, PekWY,Syap W, Johan A. Unplanned extubation: a local experience. Singapore Med J 2002;43(10):504-8.

19. Listello D, Sessler NC. Unplanned extubation clinical predictors for reintubation. Chest 1994;105(5):1496-503.

20. Krinsley JS, Barone JE. The drive to survive: unplanned extubation in the ICU. Chest 2005;128(2):560-6.

21. Whelan J, Simpson SQ, Levy H. Unplanned extubation. Predictors of successful termination of mechanical ventilatory support. Chest 1994;105(6):1808-12.

\section{Fe de errata}

En el último número de Archivos Argentinos de Pediatría, en el artículo "Consumo de tabaco en médicos residentes..." [Arch Argent Pediatr 2013;111(4):315-321], en la página 315, entre los autores ("Grupo de estudio del tabaquismo...") figura la Dra. Andrea Reinosog, cuando debería decir Dra. Andrea Reinoso ${ }^{\mathrm{h}}$ ya que su filiación corresponde al Hospital Sor María Ludovica, de la Ciudad de La Plata. 


\section{ANEXO 1. FICHA DE RECOLECCIÓN DE DATOS}

- Historia clínica:

- Nombre y apellido:

- Edad:

- Sexo:

- Motivo de ingreso a UTIP:

- PIM ll:

- $\mathrm{N}^{\circ}$ ENP:

- Fecha ENP:

- Turno de enfermería en el que ocurre la ENP:

\begin{tabular}{l|l}
\hline $6-11: 59 \mathrm{~h}$ & \\
\hline $12-17: 59 \mathrm{~h}$ & \\
\hline $18-23: 59 \mathrm{~h}$ & \\
\hline $24-5: 59 \mathrm{~h}$ & \\
\hline Franquero 0-11:59 h & \\
\hline Franquero $12-23: 59 \mathrm{~h}$ & \\
\hline
\end{tabular}

- Relación enfermero-paciente en el momento ENP:

- Nivel de sedación según el puntaje de Ramsay en el momento de la ENP:

Nivel 1 Paciente agitado, ansioso o inquieto

Nivel 2 Paciente cooperador, orientado y tranquilo

Nivel 3 Dormido con respuesta a órdenes

Nivel 4 Dormido con breve respuesta a la luz y el sonido

Nivel 5 Dormido con respuesta solo al dolor

Nivel 6 No tiene respuestas

- Factores de riesgo asociados a ENP:

\begin{tabular}{l|l|l} 
& \multicolumn{1}{c|}{ sí } & no \\
\hline Destete & & \\
\hline Soporte ventilatorio completo & & \\
\hline Transporte & & \\
\hline Fijación incorrecta del TET & & \\
\hline Secreciones abundantes & & \\
\hline Procedimiento de atención y cuidado & & \\
\hline
\end{tabular}

- Reintubación:

sí

no

- Días de ARM:

- Días Internación en UCIP:

- Egreso de UCIP:

vivo

muerto 


\section{ANEXO 2. PAQUETE DE MEDIDAS}

\section{Capacitación del personal de la UCIP}

Se dictaron clases con la finalidad de:

- Comunicar los resultados de la primera etapa de la investigación.

- Concientizar sobre la importancia y las consecuencias de la ENP.

- Difundir el contenido y la forma de implementación del paquete de medidas de mejora.

Las clases, que duraron 30 minutos y estuvieron dirigidas a todo el personal de la UCIP (médicos, enfermeros y kinesiólogos), se dictaron en todas las guardias de la semana y en todos los turnos de enfermería, y fueron de carácter obligatorio.

Para su desarrollo se usaron los siguientes elementos:

a. Presentación audiovisual con los gráficos y tablas que resumen los resultados de la investigación y el desarrollo de las medidas de mejora por implementar.

b. Video realizado en la UCIP que muestra la nueva técnica de fijación del tubo endotraqueal (TET).

Los responsables del dictado de las clases y el monitoreo de la asistencia fueron el Dr. Facundo Jorro Barón y la Dra. Claudia Meregalli. La etapa de capacitación del personal estuvo concluida cuando todo el personal de la UCIP hubo presenciado por lo menos una de las clases. La información se registró en una planilla.

\section{Estandarización de la fijación del tubo endotraqueal}

Primer paso: se seleccionó el tamaño de tubo endotraqueal adecuado para la edad del paciente.

En niños mayores de 2 años se utilizaron las siguientes fórmulas:

TET sin balón (fórmula de Cole modificada):

Número de TET= edad en años $/ 4+4$

TET con balón (fórmula de Khine):

Número de TET = edad en años/4 +3

En los niños menores de 2 años, al no haber una fórmula para calcular el tamaño del TET, este se eligió por estimación de la vía aérea según la Tabla 1.

TABLA 1. Cálculo del tamaño del tubo endotraqueal en menores de 2 años

\begin{tabular}{cc}
\hline Edad (meses) & Número de tubo endotraqueal \\
\hline$<6$ & $3,5-4$ \\
$6-24$ & $4-4,5$ \\
\hline
\end{tabular}

Segundo paso: se determinó la profundidad a la cual se fijaría el TET.

La profundidad del TET se calculó con la siguiente fórmula:

Profundidad TET $(\mathrm{cm})=$ número de TET (según formula correspondiente) $\times 3$

Tercer paso: se fijó el TET. El responsable de la fijación del TET fue el kinesiólogo de guardia y enfermero a cargo del paciente, utilizando Tensoplast Sport $^{\circledR}$.

Técnica de fijación:

1) Cortar una tira de Tensoplast Sport ${ }^{\circledR}$.

2) Secar la zona de la cara y el TET con gasa.

3) Extender una de las tiras de Tensoplast Sport ${ }^{\circledR}$ por encima del labio superior hasta el centro de este.

4) Sujetar el TET pasando la tira a su alrededor dos veces.

5) Continuar la fijación por encima del labio superior en el lado opuesto.

Cuarto paso: se verificó por radiografía de tórax de frente la profundidad del TET. Este debe estar a la altura de la segunda vértebra dorsal a por lo menos $0,5 \mathrm{~cm}$ de la carina. 
Quinto paso: confirmada la correcta colocación del TET, se confeccionó una ficha.

La ficha contenía la siguiente información:

- la identificación del paciente

- el número de TET

- la profundidad del TET

Se colgó la ficha a la cabecera del paciente.

Esta información también se registró en la hoja de prácticas de enfermería.

3. Estandarización del monitoreo de la fijación del TET:

Se verificó la correcta fijación del TET una vez por turno de enfermería y antes de la higiene del paciente. El responsable del control fue el enfermero a cargo del paciente. La información se registró en la hoja de prácticas de enfermería.

La fijación se cambió en los siguientes casos:

- Fijación floja o sucia.

- Necesidad de modificar la profundidad del TET. 